\title{
The Spectrum of NF1 Gene Variations in Southeastern Turkey
}

\author{
(D) Emre Kırat1, (D) Hatice Mutlu Albayrak² \\ ${ }^{1}$ Gaziantep Ersin Arslan Training and Research Hospital, Clinic of Medical Genetics, Gaziantep, Turkey \\ ²Cengiz Gökçek Maternity and Children's Hospital, Clinic of Pediatric Genetics, Gaziantep, Turkey
}

\begin{abstract}
Aim: We aimed to expand the variant spectrum of the NF1 gene in Southeastern Turkey. Neurofibromatosis type 1 (NF1) disease is an inherited skin disorder with variable severity and heterogeneous systemic involvement. The pathogenic variations of the NF1 gene are responsible for the NF1 phenotype.

Materials and Methods: In this study, clinical and molecular manifestations of 92 molecularly confirmed NF1 patients from 86 unrelated families are presented. The next-generation sequencing method (using lon Torrent PGM ${ }^{\mathrm{m}}$ Platform) was performed to analyze all coding exons of the NF1 gene.

Results: Seventy-six different NF7 variations were identified with 27 of them being novel. $42.5 \%$ of the patients were familial and $57.5 \%$ were sporadic. Except for one 20-year-old patient with c.1637dupT variant who presented with pilocytic astrocytoma without cutaneous findings, all the other patients demonstrated several typical clinical criteria of NF1.

Conclusion: Although NF1 diagnostic criteria are the most widely used and proficient clinical diagnostic tool, NF1 gene analysis can be applied as a definitive diagnostic tool in cases with atypical presentations and in early childhood.
\end{abstract}

Keywords: NF1, next-generation sequencing, Southeastern Turkey

\section{Introduction}

Neurofibromatosis type 1 (NF1; OMIM 162200) is one of the most common hereditary disorders. It is inherited as an autosomal dominant trait. The estimated incidence at birth is $1 / 3,000(1,2)$. Multiple café-au-lait spots, axillary/ inguinal freckling, multiple cutaneous neurofibromas, iris Lisch nodules, and choroidal freckling are the characteristic features of NF1 (3). NF1 also manifests as multisystemic disorders with musculoskeletal, vascular, central nervous, and peripheral nervous system involvement such as scoliosis, tibial dysplasia, vasculopathy, glioma, and malignant peripheral nerve sheath tumors (4).
Clinical diagnosis of NF1 is based on the National Institutes of Health (NIH) diagnostic criteria (5). However, without a family history, these criteria may be insufficient in early childhood as most of the clinical features manifest later in life (3).

The NF1 phenotype is caused by germline heterozygous pathogenic variants of the NF1 gene. NF1 is located at chromosome 17q11.2 and coded neurofibromin 1 protein that acts as a regulator of Ras activity. NF1, one of the largest genes in the human genome, consists of 57 coding exons and 12,362 base pairs transcript length (transcript reference, NM_000267.3).

\section{Address for Correspondence}

Hatice Mutlu Albayrak, Cengiz Gökçek Maternity and Children's Hospital, Clinic of Pediatric Cenetics, Gaziantep, Turkey Phone: +90 5063705032 E-mail: haticemutlu@gmail.com ORCID: orcid.org/0000-0001-5624-3878

Received: 27.10 .2020 Accepted: 12.01 .2021

${ }^{\circ}$ Copyright 2021 by Ege University Faculty of Medicine, Department of Pediatrics and Ege Children's Foundation The Journal of Pediatric Research, published by Galenos Publishing House. 
In recent years, next-generation sequencing (NGS) technology has enabled many genes, regardless of size, to be analyzed systematically, comprehensively, more easily and more cost-effectively. Most studies demonstrated that NGS with in-solution hybridization-based enrichment

provides a high mutation detection rate comparable to that of conventional direct capillary sequencing methods for the molecular diagnosis of neurofibromatosis. In this study, we aimed to present the variant spectrum of NF1 patients from the region of Southeastern Turkey and investigate if there is a clear genotype-phenotype correlation (6).

\section{Materials and Methods}

\section{Profile of the Patients}

The clinical and genetic data of the NF1 patients who were referred to the Medical Genetics Clinic, Gaziantep Ersin Arslan Training and Research Hospital between May 2016 and December 2019 were evaluated retrospectively. Peripheral blood samples were obtained after taking informed consent from all participants or the legal guardians of those children under the age of 18. The study was approved by the Ethics Committee of the Gaziantep University Medical Faculty (approval number: 65587614-774.99-291, date: 04/10/2017).

Ninety-two Turkish patients from 86 unrelated families who were both clinically and molecularly diagnosed with NF1 were included in this study. Fifty-four (58.7\%) patients were male and thirty-eight (41.3\%) were female. Age at diagnosis was in the range of 1-46 years.

The clinical diagnosis was performed based on the presence of two or more of the diagnostic criteria proposed by the NIH Consensus Development Conference (5). The diagnosis of NF1 was established in patients who had two or more of the following $\mathrm{NIH}$ criteria: Six or more cafeau-lait macules (one of them must be greater than $5 \mathrm{~mm}$ and $15 \mathrm{~mm}$, prepubertal and post-pubertal respectively); two or more neurofibromas or 1 plexiform neurofibroma; freckling in the axillary or inguinal regions; optic glioma; two or more Lisch nodules; a distinctive osseous lesion (sphenoid dysplasia or tibial pseudarthrosis); and a firstdegree relative with neurofibromatosis type 1.

\section{Genetic Analysis}

Genomic DNA was extracted from whole blood samples using an automated method (RSC whole blood DNA kit) in the Maxwell ${ }^{\circledR} 16$ (Promega Corporation, Madison, WI). NF1 (57 coding exons, NM_000267.3) amplicons were designed using the AmpliSeq Designer software (Life Technologies, (A, USA), targeting the complete coding sequence of the NF1 gene by 120 amplicons. The design target coverage was $99.49 \%$.

Amplicon library was prepared using the Ion AmpliSeq Library Kit Plus, Xpress Barcode Adapters 1-96 Kit (Thermo Fisher Scientific), then pooled together using Qubit 1X dsDNAhs Assay kit and Qubit 4 Fluorometer (Thermo Fisher Scientific). Emulsion PCR, and Ion Sphere Particles enrichment were carried out in the Ion Chef System, then loaded into an Ion 530 chip. NGS was performed via Ion 510 \& Ion 520 \& Ion 530 kit-Chef (Thermo Fisher Scientific). Data were processed using Ion Torrent Suite Software (Thermo Fisher Scientific) and Ion Reporter Software (Thermo Fisher Scientific).

The Human Gene Mutation Database (HCMD) (7) and Leiden Open Variation Database (LOVD) were used to determine whether a variant was novel or not. Several prediction algorithms, including SIFT (http://sift.jcvi. org), Polyphen2 (http://genetics.bwh.harvard.edu/pph2/), Human Splicing Finder (http://www.umd.be/HSF/) and Mutation taster (http://www.mutationtaster.org) were used to determine any damaging effects on the protein. The Genome Aggregation Database (https://gnomad. broadinstitute.org) was used to estimate the minor allele frequency score.

Nomenclature of the variants was based on the NM_000267.3 (NCBI transcript number). Variants were reviewed using dbSNP (http://www.ncbi.nlm.nih.gov/ projects/SNP/). A minimum 30X coverage for all bases was accepted for a reliable variant calling. Ion Reporter version 5.0 software was used to annotate variants. Integrated Genomics Viewer (http://software.broadinstitute.org/ software/igv/) was used for visual assessment of the revealed variants.

Novel variants are reported to the Human Genome Variation Society guidelines and checked by using Mutalyzer tool (https://mutalyzer.nl/about). All variants were classified by using The American College of Medical Genetics and Genomics (ACMG) guidelines criteria (8). Some variants were validated with direct capillary sequencing that was performed by using the BigDyeTerminator kit v3.1 (LifeTechnologies, Darmstadt, Germany) and an automated capillary sequencer $(3500 x \mathrm{x}$ Genetic Analyzer, Life Technologies). The obtained sequence data were analyzed using the Seq-Scape (Ver. 2.1) program (Applied Biosystems). 


\section{Results}

\section{Clinical Manifestations of the Patients}

We reviewed the clinical data in 73 of the 92 patients. The frequencies of clinical features are sorted by age ranges in Table I. The median age was 8 years. $42.5 \%$ of patients were familial and $57.5 \%$ were sporadic cases. Seventy-one (97.2\%) patients were suffering from café-au-lait spots. Axillary or inguinal freckling was present in 27 patients (36.9\%). The other common skin manifestation were cutaneous neurofibromas that accounted for 19.1\% (14/73) of cases. Optic glioma was identified in $6.8 \%(5 / 73)$ of the patients. Hamartomas were detected with magnetic resonance imaging in 22 of the patients (30.1\%) (Table II).

\section{Characterization of the NF1 Variants}

Seventy-six variations of which two were probably somatic were detected in 92 patients from unrelated families. The identified variants were as follows: 36 frameshift variants (41.9\%) resulting from small insertions, deletions or indels; 27 non-sense variations (31.4\%); 14 missense variants (16.2\%); 6 splicing alterations (7\%), and 3 in-frame deletions or indels (3.5\%) (Figure 1).

Twenty-seven (35\%) of the variants were novel and had not been previously reported in HGMD or LOVD. The c.2446C > T, c.3826C >T, c.5839C > T, c.2546dupC showed familial segregation. The c.5107C > T (15\%), c.3721C>T (20\%) variants were detected at lower fraction percentages; $15 \%$ and $20 \%$ respectively. The c.2033dupC, c.2446C $>T$, c.3525_3526delAA, c.3826C $>T$, c.4084C $>T, \quad$ c.5546C $>$ A, c. $6792 C>C$ variations were identified in more than one unrelated family (Table III). Only one of the variations (c.7674G >A) was interpreted as a variant of unknown origin (VUS) based on ACMG criteria. The other three were likely pathogenic and 23 of them were pathogenic. 24 variants produced truncated protein as a result of premature stop codon, which is a significant indication of their pathogenicity. All novel variants were predicted to be deleterious by at least one in-silico analysis.

\section{Discussion}

As NF1 is the one of the most common inherited disorders and NF1 is one of the largest genes in the human genome, a great number of variations (over 3,000 ) have been reported in HGMD to date. Even though previously reported pathogenic variation types show diversity, most of them cause severe truncated gene products (9). Most of the pathogenic variants (93\%) are small nucleotide alterations (including non-sense, missense, insertion or deletion) and splicing variants. Intragenic deletions/duplications (2\%) and microdeletions (5\%) are rarely detected (10). In this study, we identified 76 different mutations in 92 families. Most of the variant types are frameshift, non-sense and splicesite, similar to the literature (10-12). The missense variation rate was relatively high (14 variants, $16 \%$ ) in accordance with similar studies (11-13). There were some conflicting pathogenicity variants such as c.7674G $>$ A and c.2764G $>A$ which were interpreted as VUS based on ACMG criteria. The patient with c.7674G >A had a typical NF1 phenotype, which was a strong indicator showing the variant's pathogenicity. However, the case with c.2764G > A variant did not reach the optimum clinical registry.

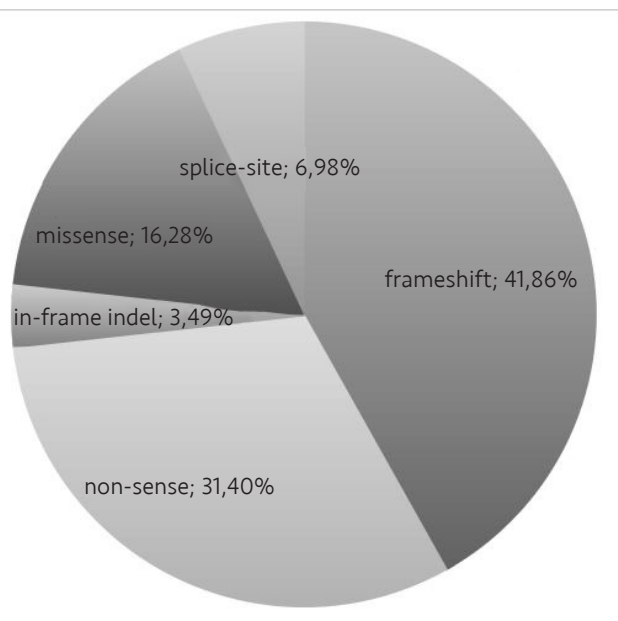

Figure 1. Type of NF1 variations

Table I. Range of the clinical features according to the age groups

\begin{tabular}{|c|c|c|c|c|c|c|c|}
\hline \multicolumn{2}{|l|}{ Age } & 6>CALs & Freckling & Neurofibroma & Optic glioma & Lisch nodule & Hamartoma \\
\hline Year & $\mathbf{n}$ & \multicolumn{6}{|l|}{ n (\%) } \\
\hline $0-1$ & 15 & $15(100)$ & $2(13.3)$ & $2(13.3)$ & $2(13.3)$ & $5(33.3)$ & $4(26.6)$ \\
\hline $2-4$ & 13 & $13(100)$ & $4(30.7)$ & $1(7.6)$ & $2(15.3)$ & $4(30.7)$ & $5(38.4)$ \\
\hline $5-18$ & 34 & $34(100)$ & $15(100)$ & $4(11.7)$ & $1(12.9)$ & $6(17.6)$ & $12(35.2)$ \\
\hline $19-30$ & 4 & $3(75)$ & $3(44.1)$ & $1(25)$ & $0(0)$ & $0(0)$ & $0(0)$ \\
\hline $31-60$ & 7 & $6(85.7)$ & $3(75)$ & $6(87.5)$ & $0(0)$ & $2(28.5)$ & $1(14.2)$ \\
\hline
\end{tabular}




\begin{tabular}{|c|c|c|c|c|c|c|c|c|c|c|c|c|}
\hline Family & Case & Sex & $\begin{array}{l}\text { Age } \\
\text { (yr) }\end{array}$ & $\begin{array}{l}\text { Variation (codon } \\
\text { number) }\end{array}$ & $\begin{array}{l}\text { Family } \\
\text { history }\end{array}$ & CALs & Neurofibromas & Freckling & $\begin{array}{l}\text { Optic } \\
\text { glioma }\end{array}$ & $\begin{array}{l}\text { Lisch } \\
\text { nodules }\end{array}$ & Hamartoma & $\begin{array}{l}\text { Other } \\
\text { clinical } \\
\text { findings }\end{array}$ \\
\hline F1 & $\mathrm{C} 1$ & M & 1 & c.1756_1759del & - & + & - & - & - & - & $N / R$ & - \\
\hline F2 & $\mathrm{C} 2$ & M & 13 & c. $5546 \mathrm{G}>\mathrm{A}$ & + & + & - & - & - & - & + & - \\
\hline F3 & C3 & M & 15 & c.3525_3526delAA & $N / R$ & $N / R$ & $N / R$ & $N / R$ & $N / R$ & $N / R$ & + & $N / R$ \\
\hline $\mathrm{F} 4$ & C4 & $\mathrm{F}$ & 1 & c.3739delT & - & + & - & - & + & - & + & - \\
\hline F5 & C5 & $\mathrm{F}$ & 11 & c.1019_1020delCT & $N / R$ & $N / R$ & $N / R$ & $N / R$ & $N / R$ & $N / R$ & $N / R$ & $N / R$ \\
\hline F6 & C6 & M & 2 & c.1393-9T>A & + & + & - & $N / R$ & - & - & - & $N / R$ \\
\hline F7 & C7 & $\mathrm{F}$ & 1 & c.1428_1431delATTTinsCC & + & + & - & $N / R$ & - & - & - & - \\
\hline F8 & C8 & M & 7 & c. $1466 \mathrm{~A}>\mathrm{C}$ & - & $N / R$ & - & - & - & - & - & - \\
\hline F9 & C9 & $\mathrm{F}$ & 13 & c.1557dupA & + & + & - & $N / R$ & - & - & $N / R$ & - \\
\hline F10 & $\mathrm{C} 10$ & M & 45 & c.1697delC & - & + & + & $N / R$ & $N / R$ & $N / R$ & - & - \\
\hline F11 & C11 & M & 41 & c. $1721+3 A>C$ & + & - & + & $N / R$ & $N / R$ & $N / R$ & $N / R$ & - \\
\hline F12 & C12 & $\mathrm{F}$ & 11 & c. $2446 C>T$ & + & + & + & + & - & - & + & - \\
\hline F12 & C13 & M & 6 & c. $2446 C>T$ & + & + & $N / R$ & - & $N / R$ & $N / R$ & - & $\begin{array}{l}\text { Short } \\
\text { stature }\end{array}$ \\
\hline $\mathrm{F} 12$ & C14 & $\mathrm{F}$ & 28 & c. $2446 C>T$ & + & + & - & + & $N / R$ & $\mathrm{~N} / \mathrm{R}$ & - & - \\
\hline F13 & C15 & $\mathrm{F}$ & 11 & c. $2446 C>T$ & - & + & + & + & - & - & $N / R$ & - \\
\hline F14 & $\mathrm{C} 16$ & $\mathrm{~F}$ & 11 & c.2970_2972delAAT & - & + & - & $N / R$ & - & - & - & - \\
\hline F15 & C17 & M & 1 & c. $3826 C>T$ & - & + & + & $N / R$ & + & + & + & Seizure \\
\hline F16 & C18 & $\mathrm{F}$ & 2 & c. $3826 C>T$ & + & + & - & - & - & - & - & - \\
\hline F16 & C19 & $\mathrm{F}$ & 1 & c. $3826 C>T$ & + & + & - & - & - & + & - & - \\
\hline F16 & $\mathrm{C} 20$ & M & 38 & c. $3826 C>T$ & + & + & + & + & - & + & $\mathrm{N} / \mathrm{R}$ & - \\
\hline F17 & C21 & $\mathrm{F}$ & 8 & c.3916C>T & - & + & - & + & - & + & + & - \\
\hline F18 & $\mathrm{C} 22$ & M & 10 & c.4075_4076delinsAA & $N / R$ & $N / R$ & $N / R$ & $N / R$ & $N / R$ & $N / R$ & $\mathrm{~N} / \mathrm{R}$ & $N / R$ \\
\hline F19 & $\mathrm{C} 23$ & $\mathrm{~F}$ & 9 & c. $4084 C>T$ & + & + & + & + & - & + & + & Seizure \\
\hline F20 & C24 & M & 35 & c. $4084 C>T$ & - & + & + & - & - & + & - & - \\
\hline F21 & $\mathrm{C} 25$ & $\mathrm{~F}$ & 9 & c. $4267 A>C$ & - & + & - & + & + & + & - & Asthma \\
\hline F22 & $\mathrm{C} 26$ & M & 35 & c. $4537 C>T$ & + & + & - & + & $N / R$ & - & $N / R$ & Arrhythmia \\
\hline F23 & $\mathrm{C} 27$ & M & 9 & c.4621delC & - & + & - & + & - & + & + & - \\
\hline F24 & $\mathrm{C} 28$ & $\mathrm{~F}$ & 2 & c.4822_4826delCTGAC & + & + & $N / R$ & + & - & - & - & - \\
\hline F25 & C29 & M & 5 & c. $5522 \mathrm{~T}>\mathrm{A}$ & - & + & - & + & - & + & - & $N / R$ \\
\hline F26 & C30 & M & 1 & $c .5722 \mathrm{G}>\mathrm{T}$ & - & + & - & + & $N / R$ & + & $N / R$ & - \\
\hline F27 & C31 & M & 12 & c. $5839 \mathrm{C}>\mathrm{T}$ & + & + & - & + & - & + & + & $N / R$ \\
\hline F27 & C32 & $\mathrm{F}$ & 8 & c. $5839 \mathrm{C}>\mathrm{T}$ & + & + & - & + & - & - & $\mathrm{N} / \mathrm{R}$ & - \\
\hline F28 & C33 & M & 3 & c.6334_6335delCT & - & + & - & - & - & - & - & $N / R$ \\
\hline F29 & C34 & M & 1 & c.6791dupA & - & + & - & - & - & + & + & - \\
\hline $\mathrm{F} 30$ & C35 & M & 1 & c. $6792 C>C$ & - & + & - & - & - & - & - & $N / R$ \\
\hline F31 & C36 & $\mathrm{F}$ & 13 & c. $6792 C>C$ & - & + & - & + & - & - & + & - \\
\hline F32 & C 37 & $\mathrm{~F}$ & 10 & c. $6792 C>C$ & $N / R$ & $N / R$ & $N / R$ & $N / R$ & $N / R$ & $N / R$ & $N / R$ & $N / R$ \\
\hline
\end{tabular}


Table II. continued

\begin{tabular}{|c|c|c|c|c|c|c|c|c|c|c|c|c|}
\hline F33 & C38 & $\mathrm{F}$ & 5 & c.7206_7207delCA & $N / R$ & $N / R$ & $N / R$ & $N / R$ & $N / R$ & $N / R$ & $N / R$ & $N / R$ \\
\hline F34 & C39 & M & 25 & c. $7237 C>T$ & - & + & - & + & - & - & - & - \\
\hline F35 & C40 & M & 10 & c. $7285 C>T$ & - & + & - & - & - & - & - & - \\
\hline F36 & C41 & M & 29 & c.7419G $>$ A & + & + & + & + & - & - & - & - \\
\hline F37 & C42 & M & 9 & c. $7486 C>T$ & - & + & $N / R$ & $N / R$ & - & - & - & $N / R$ \\
\hline F38 & C43 & $\mathrm{F}$ & 9 & c.953_956delAAAG & - & + & - & $N / R$ & $N / R$ & $N / R$ & - & Scoliosis \\
\hline F39 & C44 & M & 10 & c.1548dupC & + & + & - & yok & - & - & - & - \\
\hline F40 & C45 & M & 13 & - & - & + & - & $N / R$ & - & - & + & - \\
\hline F41 & C46 & M & 46 & c.2867_2868delCCinsA & $N / R$ & $N / R$ & $N / R$ & $N / R$ & $N / R$ & $N / R$ & $N / R$ & $N / R$ \\
\hline F42 & C47 & M & 3 & c.2890dupA & $N / R$ & $N / R$ & $N / R$ & $N / R$ & $N / R$ & $N / R$ & $N / R$ & $N / R$ \\
\hline F43 & C48 & $\mathrm{F}$ & 1 & c.3457_3460delCTCA & - & + & - & - & - & - & - & $N / R$ \\
\hline F44 & C49 & $\mathrm{F}$ & 2 & c.3525_3526delAA & + & + & - & - & - & - & - & $N / R$ \\
\hline F45 & C50 & M & 8 & c.5844_5845delAA & - & + & - & + & - & - & - & $N / R$ \\
\hline F46 & C51 & M & 4 & c.7674G $>A$ & - & + & - & + & - & + & + & - \\
\hline F47 & C52 & $\mathrm{F}$ & 3 & c.2033dupC & + & + & $N / R$ & $N / R$ & - & - & $N / R$ & - \\
\hline F48 & C53 & $\mathrm{F}$ & 7 & c. $1381 C>T$ & - & + & - & $N / R$ & - & - & + & - \\
\hline F49 & C54 & M & 4 & $\begin{array}{l}\text { c.1261-3 1270del } \\
\text { TAGTCCCCATTCC }\end{array}$ & $N / R$ & $N / R$ & $N / R$ & $N / R$ & $N / R$ & $N / R$ & $N / R$ & $N / R$ \\
\hline F50 & C55 & $\mathrm{F}$ & 3 & c. $1318 \mathrm{C}>\mathrm{T}$ & - & + & - & + & - & - & + & - \\
\hline F51 & $C 56$ & $\mathrm{~F}$ & 16 & c. $1721 \mathrm{G}>\mathrm{A}$ & $N / R$ & $N / R$ & $N / R$ & $N / R$ & + & $N / R$ & - & $N / R$ \\
\hline F52 & C57 & M & 8 & c.2546dupG & + & + & - & - & - & + & - & - \\
\hline F52 & C58 & M & 1 & c.2546dupG & + & + & + & + & - & + & + & - \\
\hline F53 & C59 & $F$ & 2 & c.3058delG & + & + & - & + & + & - & + & $\begin{array}{l}\text { Skeletal } \\
\text { deformity }\end{array}$ \\
\hline F54 & C60 & $\mathrm{F}$ & 13 & c. $3114-2 A>C$ & - & + & - & - & - & - & - & - \\
\hline F55 & C61 & M & 6 & c. $3610 C>C$ & - & + & - & - & - & - & + & Seizure \\
\hline F56 & C62 & M & 8 & c.5003_5004insCGTA & $N / R$ & $N / R$ & $N / R$ & $N / R$ & $N / R$ & $N / R$ & $N / R$ & $N / R$ \\
\hline F57 & C63 & M & 3 & c.6263delT & $N / R$ & $N / R$ & $N / R$ & $N / R$ & $N / R$ & $N / R$ & $N / R$ & $N / R$ \\
\hline F58 & C64 & M & 8 & c. $4288 \mathrm{~A}>\mathrm{C}$ & - & + & - & + & - & - & $N / R$ & $N / R$ \\
\hline F59 & C65 & M & 36 & c.6125delT & + & + & + & + & - & - & + & - \\
\hline F60 & C66 & M & 12 & c. $1496 \mathrm{~T}>\mathrm{C}$ & + & + & - & $N / R$ & - & - & - & - \\
\hline F61 & C67 & $\mathrm{F}$ & 4 & c.1737_1738delTT & + & + & $1+$ & - & - & + & - & - \\
\hline $\mathrm{F} 62$ & C68 & M & 21 & c. $1748 \mathrm{~A}>\mathrm{C}$ & $N / R$ & N/R & $N / R$ & $N / R$ & $\mathrm{~N} / \mathrm{R}$ & $N / R$ & $N / R$ & $\mathrm{~N} / \mathrm{R}$ \\
\hline F63 & C69 & $\mathrm{F}$ & 6 & c. $1885 \mathrm{G}>\mathrm{A}$ & + & + & - & $N / R$ & - & - & - & - \\
\hline F64 & C70 & $\mathrm{F}$ & 16 & c.2033dupC & + & + & $N / R$ & $N / R$ & $\mathrm{~N} / \mathrm{R}$ & $N / R$ & $N / R$ & $N / R$ \\
\hline F65 & C71 & M & 13 & c.2033dupC & + & + & - & + & - & - & - & - \\
\hline F66 & C72 & $\mathrm{F}$ & 13 & c.2097dupC & $N / R$ & $N / R$ & $N / R$ & $N / R$ & $\mathrm{~N} / \mathrm{R}$ & $N / R$ & $N / R$ & $N / R$ \\
\hline F67 & $\mathrm{C} 73$ & M & 9 & c. $2252-3 T>C$ & + & + & $N / R$ & $N / R$ & $N / R$ & $N / R$ & + & Seizure \\
\hline F68 & C74 & M & 1 & c. $2325+3 A>C$ & - & + & - & - & - & - & - & $N / R$ \\
\hline F69 & C75 & $\mathrm{F}$ & 1 & c.2604delT & $N / R$ & $N / R$ & $N / R$ & $N / R$ & $N / R$ & $N / R$ & $N / R$ & $N / R$ \\
\hline F70 & C76 & M & 5 & c. $2764 \mathrm{C}>\mathrm{A}$ & $N / R$ & $N / R$ & $N / R$ & $N / R$ & $N / R$ & $N / R$ & $N / R$ & $N / R$ \\
\hline F71 & $\mathrm{C} 77$ & $\mathrm{~F}$ & 13 & c.3470T $>A$ & - & + & $N / R$ & $N / R$ & $N / R$ & $N / R$ & $N / R$ & $N / R$ \\
\hline
\end{tabular}


Table II. continued

\begin{tabular}{|c|c|c|c|c|c|c|c|c|c|c|c|c|}
\hline F72 & $\mathrm{C} 78$ & M & 2 & c.446dupA & - & + & $N / R$ & - & + & + & + & $\begin{array}{l}\text { Skeletal } \\
\text { deformity }\end{array}$ \\
\hline F73 & C79 & M & 10 & c. $484 C>T$ & $N / R$ & $N / R$ & $N / R$ & $N / R$ & $N / R$ & $N / R$ & $N / R$ & $N / R$ \\
\hline F74 & C80 & $\mathrm{F}$ & 9 & c. $4817 \mathrm{~T}>\mathrm{A}$ & + & + & - & + & - & - & - & Scoliosis \\
\hline F75 & C81 & $\mathrm{F}$ & 1 & c.5719G >T & - & + & - & - & - & - & - & - \\
\hline F76 & C82 & M & 1 & c.7229delT & - & + & - & - & - & - & - & - \\
\hline F77 & C83 & M & 2 & c.7518_7519delCCinsCT & + & + & - & - & - & - & - & - \\
\hline F78 & C84 & $\mathrm{F}$ & 1 & c.1404dupT & - & + & - & - & $N / R$ & $N / R$ & - & - \\
\hline F79 & C85 & M & 20 & c.1637dupT & - & - & - & - & - & - & - & $\begin{array}{l}\text { Pilositic } \\
\text { astrocytoma }\end{array}$ \\
\hline F80 & C86 & $\mathrm{F}$ & 13 & c.1756_1759delACTA & - & + & +1 & + & - & - & $N / R$ & - \\
\hline F81 & C87 & M & 21 & c. $3721 C>T$ & N/R & $\mathrm{N} / \mathrm{R}$ & $\mathrm{N} / \mathrm{R}$ & N/R & $N / R$ & $N / R$ & - & $N / R$ \\
\hline F82 & $\mathrm{C} 88$ & $\mathrm{~F}$ & 8 & c.3871delG & $N / R$ & $N / R$ & $N / R$ & $N / R$ & $N / R$ & $N / R$ & $N / R$ & $N / R$ \\
\hline F83 & C89 & M & 7 & c.4816_4817insGG & $N / R$ & + & - & - & - & - & + & $N / R$ \\
\hline F84 & $C 90$ & M & 1 & c. $5107 C>T$ & - & + & - & - & - & - & $N / R$ & - \\
\hline F85 & C91 & M & 4 & c. $5546 \mathrm{G}>\mathrm{A}$ & N/R & + & - & $N / R$ & - & + & + & Speech delay \\
\hline F86 & C92 & M & 38 & c.5827delG & - & + & + & - & - & - & - & - \\
\hline
\end{tabular}

There is not a well-recognized hot spot region in the NF1 gene (14-16). In this study, we found only one recurrent variant (c.6792C>C) in two unrelated families. It did not show significant variant aggregation in any exon (Figure 2). Although we observed relative variant density in exon 13 according to the exon length (Figure 2), it was insufficient to speculate that exon 13 is a hotspot region. Moreover, the variant frequency was lower both in the first and last few exons which code the constitutional amino acids. This can be attributed to the variants on the distal part of the gene being less effective in causing the NF1 phenotype.

Six functional domains were determined on the NFI protein: The cysteine and serine rich domain (CSRD; exons 1-22), the tubulin binging domain (TBD; exons 22-27), the domain responsible for interactions with RAS and CTP hydrolysis (GRD; exons 27-34), the bipartite lipid binging domain (first part) (Sec14; exons 35-36), the bipartite lipid binging domain (second part) ( $\mathrm{PH}$; exons 35-36), and the carboxyl-terminal domain (CTD; exons 37-52) (17). The distribution of the variants according to functional domains were CSRD (31/76-40.7\%), TBD (9/76-11.8\%), GRD (11/76$14.4 \%)$ Sec 14 and PH (5/76-6.5\%), and CTD (20/76-26.3\%). We did not find any variants outside of the functional domains.



Figure 2. Distribution of the variants according to functional domains 


\begin{tabular}{|c|c|c|c|c|c|c|c|c|c|}
\hline Family & $\begin{array}{l}\text { Variation (codon } \\
\text { number) }\end{array}$ & Protein number & $\begin{array}{l}\text { HGMD } \\
\text { reference }\end{array}$ & LOVD & Type & $\begin{array}{l}\text { Predicted } \\
\text { effect }\end{array}$ & $\begin{array}{l}\text { ACMG } \\
\text { criteria }\end{array}$ & $\begin{array}{l}\text { ACMG } \\
\text { prediction }\end{array}$ & Exon \\
\hline F1, F80 & c.1756_1759del & p.Thr586Valfs*18 & CD982825 & $\mathrm{R}$ & Deletion & Frameshift & $\begin{array}{l}\text { PVS1, PM1, } \\
\text { PM2 PP3, } \\
\text { PP5 }\end{array}$ & $P$ & 16 \\
\hline$F 2$, F85 & c. $5546 \mathrm{G}>\mathrm{A}$ & p.Arg1849Gln & CM1718194 & $\mathrm{R}$ & Substitution & Missense & $\begin{array}{l}\text { PM2, PP2, } \\
\text { PP3, PP5 }\end{array}$ & LP & 37 \\
\hline F3, F44 & c.3525_3526delAA & p.Arg1176Serfs*18 & CD000971 & $\mathrm{R}$ & Deletion & Frameshift & $\begin{array}{l}\text { PVS1, PM1, } \\
\text { PM2, PP3, } \\
\text { PP5 }\end{array}$ & $P$ & 27 \\
\hline F4 & c.3739delT & p.Phe1247Leufs*19 & Novel & $N / R$ & Deletion & Frameshift & $\begin{array}{l}\text { PVS1, PM1, } \\
\text { PM2, PP3 }\end{array}$ & $P$ & 28 \\
\hline F5 & c.1019_1020delCT & p.Ser340fs & CD972347 & $\mathrm{R}$ & Deletion & Frameshift & $\begin{array}{l}\text { PVS1, PM1, } \\
\text { PM2, PP3, } \\
\text { PP5 }\end{array}$ & $P$ & 9 \\
\hline F6 & c.1393-9T>A & - & CSO00058 & $R(P)$ & Substitution & Splice site & PM2 & VUS & $12-13$ \\
\hline F7 & c.1428_1431delATTTinsCC & p.Lys476Asnfs*14 & Novel & $N / R$ & Deletion & Frameshift & $\begin{array}{l}\text { PVS1, PM2, } \\
\text { PP3 }\end{array}$ & $P$ & 13 \\
\hline F8 & c. $1466 A>C$ & p.Tyr489Cys & CM1111787 & $R(P)$ & Substitution & Missense & $\begin{array}{l}\text { PM1, PM2, } \\
\text { PP2, PP3, } \\
\text { PP5 }\end{array}$ & $P$ & 13 \\
\hline F9 & c.1557dupA & p.Gly520fs & Novel & $N / R$ & Duplicaton & Frameshift & $\begin{array}{l}\text { PVS1, PM1, } \\
\text { PM2 PP3 }\end{array}$ & $P$ & 14 \\
\hline F10 & c.1697delC & p.Pro566Leufs*2 & CD1815862 & $N / R$ & Deletion & Frameshift & $\begin{array}{l}\text { PVS1, PM1, } \\
\text { PM2, PP3 }\end{array}$ & $P$ & 15 \\
\hline F11 & c. $1721+3 A>C$ & - & CS941514 & $R(P)$ & Substitution & Splice site & PM2 & VUS & $15-16$ \\
\hline $\begin{array}{l}\text { F12, } \\
\text { F13 }\end{array}$ & c. $2446 C>T$ & p.Arg816* & CM971040 & $R(P)$ & Substitution & Non-sense & $\begin{array}{l}\text { PVS1, PM1, } \\
\text { PM2, PP3, } \\
\text { PP5 }\end{array}$ & $P$ & 21 \\
\hline F14 & c.2970_2972delAAT & p.Met991del & CD931025 & $R(P)$ & Deletion & $\begin{array}{l}\text { In frame } \\
\text { deletion }\end{array}$ & $\begin{array}{l}\text { PM1, PM2, } \\
\text { PM4, PP3, } \\
\text { PP5 }\end{array}$ & $P$ & 22 \\
\hline $\begin{array}{l}\text { F15, } \\
\text { F16 }\end{array}$ & c. $3826 C>T$ & p.Arg1276* & CM950847 & $R(P)$ & Substitution & Non-sense & $\begin{array}{l}\text { PVS1, PS3, } \\
\text { PM1, PM2 } \\
\text { PP3 }\end{array}$ & $P$ & 28 \\
\hline F17 & c.3916C>T & p.Arg1306* & CM981381 & $R(P)$ & Substitution & Non-sense & $\begin{array}{l}\text { PVS1, PM1, } \\
\text { PM2, PP3, } \\
\text { PP5 }\end{array}$ & $P$ & 29 \\
\hline F18 & c.4075_4076delinsAA & p.Pro1359Asn & Novel & $N / R$ & Indel & $\begin{array}{l}\text { In-frame } \\
\text { indel }\end{array}$ & $\begin{array}{l}\text { PM1, PM2, } \\
\text { PP3, PP5 }\end{array}$ & LP & 30 \\
\hline $\begin{array}{l}\text { F19, } \\
\text { F20 }\end{array}$ & c. $4084 C>T$ & p.Arg1362* & CM971046 & $R(P)$ & Substitution & Non-sense & $\begin{array}{l}\text { PVS1, PM1, } \\
\text { PM2, PP3, } \\
\text { PP5 }\end{array}$ & $P$ & 30 \\
\hline F21 & c. $4267 A>C$ & p.Lys1423Glu & CM920506 & $R(P)$ & Substitution & Missense & $\begin{array}{l}\text { PM1, PM2, } \\
\text { PM5, PP2, } \\
\text { PP3, PP5 }\end{array}$ & $P$ & 31 \\
\hline F22 & c. $4537 C>T$ & p.Arg1513* & CM941093 & $R(P)$ & Substitution & Non-sense & $\begin{array}{l}\text { PVS1, PM1, } \\
\text { PM2, PP3, } \\
\text { PP5 }\end{array}$ & $P$ & 34 \\
\hline F23 & c.4621delC & p.Leu1541fs & Novel & $R(P)$ & Deletion & Frameshift & $\begin{array}{l}\text { PVS1, PM1, } \\
\text { PM2, PP3, } \\
\text { PP5 }\end{array}$ & $P$ & 34 \\
\hline
\end{tabular}


Table III. continued

\begin{tabular}{|c|c|c|c|c|c|c|c|c|c|}
\hline F24 & c.4822_4826delCTGAC & p.Leu1608fs & Novel & $N / R$ & Deletion & Frameshift & $\begin{array}{l}\text { PVS1, PM1, } \\
\text { PM2, PP3 }\end{array}$ & $P$ & 36 \\
\hline F25 & c. $5522 T>A$ & p.Leu1841* & Novel & $N / R$ & Substitution & Non-sense & $\begin{array}{l}\text { PVS1, PM1, } \\
\text { PM2, PP3 }\end{array}$ & $P$ & 37 \\
\hline F26 & c. $5722 \mathrm{G}>\mathrm{T}$ & p.Glu1908* & CM143452 & $R(P)$ & Substitution & Non-sense & $\begin{array}{l}\text { PVS1, PM1, } \\
\text { PM2, PP3 }\end{array}$ & $P$ & 38 \\
\hline F27 & c. $5839 C>T$ & p.Arg1947* & CM900173 & $R(P)$ & Substitution & Non-sense & $\begin{array}{l}\text { PVS1, PM1, } \\
\text { PM2, PP3, } \\
\text { PP5 }\end{array}$ & $P$ & 39 \\
\hline F28 & c.6334_6335delCT & p.Leu2112Valfs & CD1415205 & $R(P)$ & Deletion & Frameshift & $\begin{array}{l}\text { PVS1, PM1, } \\
\text { PM2, PP3, } \\
\text { PP5 }\end{array}$ & $P$ & 41 \\
\hline F29 & c.6791dupA & p.Tyr2264*fs & $\mathrm{Cl} 962317$ & $R(P)$ & Duplicaton & Frameshift & $\begin{array}{l}\text { PVS1, PM1, } \\
\text { PM2, PP3 }\end{array}$ & $P$ & 45 \\
\hline $\begin{array}{l}\text { F30, } \\
\text { F31, } \\
\text { F32 }\end{array}$ & c. $6792 C>C$ & p.Tyr2264* & CM972796 & $R(P)$ & Substitution & Non-sense & $\begin{array}{l}\text { PVS1, PM1, } \\
\text { PM2, PP3, } \\
\text { PP5 }\end{array}$ & $P$ & 45 \\
\hline F33 & c.7206_7207delCA & p.His2402Glnfs*4 & CD031873 & $R(P)$ & Deletion & Frameshift & $\begin{array}{l}\text { PVS1, PM1, } \\
\text { PM2, PP3, } \\
\text { PP5 }\end{array}$ & $\mathrm{P}$ & 48 \\
\hline F34 & c. $7237 C>T$ & p.Gln2413* & CM000817 & $N / R$ & Substitution & Non-sense & $\begin{array}{l}\text { PVS1, PM1, } \\
\text { PM2, PP3 }\end{array}$ & $P$ & 48 \\
\hline F35 & c. $7285 C>T$ & p.Arg2429* & CM000818 & $R(P)$ & Substitution & Non-sense & $\begin{array}{l}\text { PVS1, PM1, } \\
\text { PM2, PP3, } \\
\text { PP5 }\end{array}$ & $P$ & 49 \\
\hline F36 & c.7419G $>A$ & p.Trp2473* & Novel & $N / R$ & Substitution & Non-sense & $\begin{array}{l}\text { PVS1, PM1, } \\
\text { PM2, PP3, } \\
\text { PP5 }\end{array}$ & $P$ & 50 \\
\hline F37 & c. $7486 C>T$ & p.Arg2496* & CM941096 & $R(P)$ & Substitution & Non-sense & $\begin{array}{l}\text { PVS1, PM2, } \\
\text { PP3, PP5 }\end{array}$ & $P$ & 50 \\
\hline F38 & c.953_956delAAAG & p.Glu318Valfs*57 & CD1512843 & $R(P)$ & Deletion & Frameshift & $\begin{array}{l}\text { PVS1, PM1, } \\
\text { PM2, PP3 }\end{array}$ & $P$ & 9 \\
\hline F39 & c.1548dupC & p.Glu517Argfs*41 & Novel & $N / R$ & Duplicaton & Frameshift & $\begin{array}{l}\text { PVS1, PM1, } \\
\text { PM2, PP3 }\end{array}$ & $P$ & 14 \\
\hline F40 & c.2824_2833delAGCAAGTTTTinsC & - & Novel & $N / R$ & Indel & $\begin{array}{l}\text { In-frame } \\
\text { indel }\end{array}$ & $\begin{array}{l}\text { PM1, PM2, } \\
\text { PM4, PP3 }\end{array}$ & LP & 21 \\
\hline F41 & c.2867_2868delCCinsA & p.Thr956Lysfs & Novel & $N / R$ & Indel & Frameshift & $\begin{array}{l}\text { PVS1, PM1, } \\
\text { PM2, PP3 }\end{array}$ & $P$ & 22 \\
\hline F42 & c.2890dupA & p.Thr964Asnfs*11 & Novel & $N / R$ & Duplicaton & Frameshift & $\begin{array}{l}\text { PVS1, PM1, } \\
\text { PM2 PP3 }\end{array}$ & $P$ & \\
\hline F43 & c.3457_3460delCTCA & p.Leu1153Metfs & CD972351 & $R(P)$ & Deletion & Frameshift & $\begin{array}{l}\text { PVS1, PM1, } \\
\text { PM2, PP3, } \\
\text { PP5 }\end{array}$ & $P$ & 26 \\
\hline F45 & c.5844_5845delAA & p.Arg1949Serfs*6 & CD941733 & $R(P)$ & Deletion & Frameshift & $\begin{array}{l}\text { PVS1, PM1, } \\
\text { PM2, PP3, } \\
\text { PP5 }\end{array}$ & $P$ & 39 \\
\hline F46 & c.7674G $>$ A & p.Met2558lle & Novel & $N / R$ & Substitution & Missense & PM2, PP2 & VUS & 51 \\
\hline $\begin{array}{l}F 47, \\
\text { F64, } \\
\text { F65 }\end{array}$ & c.2033dupC & p.lle679Aspfs*10 & Cl951961 & $R(P)$ & Duplicaton & Frameshift & $\begin{array}{l}\text { PVS1, PM1, } \\
\text { PM2, PP3, } \\
\text { PP5 }\end{array}$ & $P$ & 18 \\
\hline F48 & c.1381C>T & p.Arg $461^{*}$ & CM000780 & $R(P)$ & Substitution & Non-sense & $\begin{array}{l}\text { PVS1, PM2, } \\
\text { PP3, PP5 }\end{array}$ & $P$ & 12 \\
\hline
\end{tabular}


Table III. continued

\begin{tabular}{|c|c|c|c|c|c|c|c|c|c|}
\hline F49 & $\begin{array}{l}\text { c.1261-3 1270del } \\
\text { TAGTCCCCATTGG }\end{array}$ & - & Novel & $N / R$ & Deletion & Splice site & $\begin{array}{l}\text { PVS1, PM2, } \\
\text { PP3 }\end{array}$ & $P$ & 12 \\
\hline F50 & c. $1318 C>T$ & p.Arg440* & CM950845 & $R(P)$ & Substitution & Non-sense & $\begin{array}{l}\text { PVS1, PM1, } \\
\text { PM2, PP3, } \\
\text { PP5 }\end{array}$ & $P$ & 12 \\
\hline F51 & c.1721G>A & p.Ser574Asn & CM062898 & $R(P)$ & Substitution & Missense & $\begin{array}{l}\text { PM1, PM2, } \\
\text { PM5, PP2, } \\
\text { PP3, PP5 }\end{array}$ & $P$ & 15 \\
\hline F52 & c.2546dupG & p.p.Val850Serfs*15 & Cl098059 & $R(P)$ & Duplicaton & Frameshift & $\begin{array}{l}\text { PVS1, PM1, } \\
\text { PM2, PP3 }\end{array}$ & $P$ & 21 \\
\hline F53 & c.3058delG & p.Glu1020Lysfs*2 & Novel & $N / R$ & Deletion & Frameshift & $\begin{array}{l}\text { PVS1, PM1, } \\
\text { PM2, PP3 }\end{array}$ & $P$ & 23 \\
\hline F54 & c. $3114-2 A>C$ & - & CS147208 & $R(P)$ & Substitution & Splice site & $\begin{array}{l}\text { PVS1, PM2, } \\
\text { PP3, PP5 }\end{array}$ & $P$ & $23-24$ \\
\hline F55 & c. $3610 C>C$ & p.Arg1204Gly & CM973234 & $R(P)$ & Substitution & Missense & $\begin{array}{l}\text { PM1, PM2, } \\
\text { PM5, PP2, } \\
\text { PP3, PP5 }\end{array}$ & $P$ & 27 \\
\hline F56 & c.5003_5004insGGTA & p.Tyr1668* & Novel & $N / R$ & Insertion & Non-sense & $\begin{array}{l}\text { PVS1, PM1, } \\
\text { PM2, PP3 }\end{array}$ & $P$ & 36 \\
\hline F57 & c.6263delT & p.Phe2088Serfs*2 & CD1719515 & $N / R$ & Deletion & Frameshift & $\begin{array}{l}\text { PVS1, PM1, } \\
\text { PM2, PP3, } \\
\text { PP5 }\end{array}$ & $P$ & 41 \\
\hline F58 & c. $4288 \mathrm{~A}>\mathrm{G}$ & p.Asn1430Asp & CM113590 & $R(P)$ & Substitution & Missense & $\begin{array}{l}\text { PM1, PM2, } \\
\text { PM5, PP2, } \\
\text { PP3, PP5 }\end{array}$ & $P$ & 32 \\
\hline F59 & c.6125delT & p.Leu2042Tyrfs*7 & Novel & $N / R$ & Deletion & Frameshift & $\begin{array}{l}\text { PVS1, PM2, } \\
\text { PP3 }\end{array}$ & $P$ & 41 \\
\hline F60 & c.1496T>C & p.Leu499Arg & CM1512946 & $R(P)$ & Substitution & Missense & $\begin{array}{l}\text { PM1, PM2, } \\
\text { PP2, PP3, PP5 }\end{array}$ & LP & 13 \\
\hline F61 & c.1737_1738delTT & p.Phe579Leufs*8 & Novel & $N / R$ & Deletion & Frameshift & $\begin{array}{l}\text { PVS1, PM1, } \\
\text { PM2 PP3 }\end{array}$ & $P$ & 16 \\
\hline F62 & c. $1748 A>C$ & p.Lys583Arg & CM1111788 & $R(P)$ & Substitution & Missense & $\begin{array}{l}\text { PM1, PM2, } \\
\text { PP2, PP5, BP4 }\end{array}$ & LP & 16 \\
\hline F63 & c. $1885 G>A$ & p.Gly629Arg & Novel & $R(P)$ & Substitution & Missense & $\begin{array}{l}\text { PS1, PS3, } \\
\text { PM1, PM2, } \\
\text { PP2, PP3 }\end{array}$ & $P$ & 17 \\
\hline F66 & c.2097dupC & p.Thr700Hisfs*2 & Novel & $N / R$ & Duplicaton & Frameshift & $\begin{array}{l}\text { PVS1, PM2, } \\
\text { PP3 }\end{array}$ & $P$ & 18 \\
\hline F67 & c. $2252-3 T>G$ & - & CSO86414 & $N / R$ & Substitution & Splice site & PM2, PP5 & VUS & $18-19$ \\
\hline F68 & c. $2325+3 A>C$ & - & CS1311513 & $R(P)$ & Substitution & Splice site & PM2, PP5 & VUS & $19-20$ \\
\hline F69 & c.2604delT & p.Pro869Glnfs*9 & CD153889 & $R(P)$ & Deletion & Frameshift & $\begin{array}{l}\text { PVS1, PM1, } \\
\text { PM2 }\end{array}$ & $P$ & 21 \\
\hline F70 & c. $2764 G>A$ & p.Gly922Ser & CM1719434 & $R(P)$ & Substitution & Missense & $\begin{array}{l}\text { PM2, PP2, } \\
\text { PP3 }\end{array}$ & VUS & 21 \\
\hline F71 & c. $3470 T>A$ & p.Val1157Glu & Novel & $N / R$ & Substitution & Missense & $\begin{array}{l}\text { PM1, PM2, } \\
\text { PP3, PP5 }\end{array}$ & LP & 26 \\
\hline F72 & c.446dupA & p.Asn149Lysfs*7 & Novel & $N / R$ & Duplicaton & Frameshift & $\begin{array}{l}\text { PVS1, PM2, } \\
\text { PP3 }\end{array}$ & $P$ & 4 \\
\hline F73 & c. $484 C>T$ & p.Gln162* & CM073223 & $R(P)$ & Substitution & Non-sense & $\begin{array}{l}\text { PVS1, PM1, } \\
\text { PM2 PP3, PP5 }\end{array}$ & $P$ & 5 \\
\hline
\end{tabular}


Table III. continued

\begin{tabular}{|c|c|c|c|c|c|c|c|c|c|}
\hline F74 & c. $4817 \mathrm{~T}>\mathrm{A}$ & p.Val1606Asp & CM1919720 & $N / R$ & Substitution & Missense & $\begin{array}{l}\text { PM1, PM2, } \\
\text { PP3, PP5 }\end{array}$ & LP & 36 \\
\hline F75 & c. $5719 \mathrm{G}>\mathrm{T}$ & p.Glu1907* & CM043552 & $N / R$ & Substitution & Non-sense & $\begin{array}{l}\text { PVS1, PM1, } \\
\text { PM2 PP3, PP5 }\end{array}$ & $P$ & 38 \\
\hline F76 & c.7229delT & p.Val2410Glyfs*9 & Novel & $N / R$ & Deletion & Frameshift & $\begin{array}{l}\text { PVS1, PM1, } \\
\text { PM2 }\end{array}$ & $P$ & 48 \\
\hline F77 & c.7518_7519delGCinsCT & p.Gln $2507^{*}$ & Novel & $N / R$ & Indel & Non-sense & $\begin{array}{l}\text { PVS1, PM1, } \\
\text { PM2 PP3 }\end{array}$ & $P$ & 50 \\
\hline F78 & c.1404dupT & p.Lys469* & Novel & $N / R$ & Duplicaton & Non-sense & $\begin{array}{l}\text { PVS1, PM1, } \\
\text { PM2 PP3 }\end{array}$ & $P$ & 13 \\
\hline F79 & c.1637dupT & p.Met546llefs*12 & Novel & $N / R$ & Duplicaton & Frameshift & $\begin{array}{l}\text { PVS1, PM2, } \\
\text { PP3 }\end{array}$ & $P$ & 14 \\
\hline F81 & c. $3721 C>T$ & p.Arg1241* (\%20) & CM000799 & $R(P)$ & Substitution & Non-sense & $\begin{array}{l}\text { PVS1, PM1, } \\
\text { PM2, PP3, } \\
\text { PP5 }\end{array}$ & $P$ & 28 \\
\hline F82 & c.3871delG & p.Val1291Tyrfs*18 & Novel & $N / R$ & Deletion & Frameshift & $\begin{array}{l}\text { PVS1, PM1, } \\
\text { PM2, PP3 }\end{array}$ & $P$ & 29 \\
\hline F83 & c.4816_4817insGG & p.Val1606Glyfs*4, & Novel & $N / R$ & Insertion & Frameshift & $\begin{array}{l}\text { PVS1, PM1, } \\
\text { PM2, PP3 }\end{array}$ & $P$ & 36 \\
\hline F84 & c. $5107 C>T$ & p.Gln1703*(\%15) & CM143429 & $R(P)$ & Substitution & Non-sense & $\begin{array}{l}\text { PVS1, PM1, } \\
\text { PM2, PP3 }\end{array}$ & $P$ & 36 \\
\hline F86 & c.5827delC & p.Asp1943Metfs*15 & Novel & $N / R$ & Deletion & Frameshift & $\begin{array}{l}\text { PVS1, PM1, } \\
\text { PM2, PP3 }\end{array}$ & $P$ & 39 \\
\hline
\end{tabular}

Our results did not reveal any clear relationships between specific NF1 variants and phenotypes. Furthermore, no complete well-known genotype-phenotype correlation has been reported in the literature to date $(12,18-20)$. Only three clear correlations of clinical significance have been identified in particular pathogenic NF1 variants. NF1 wholegene deletions are related with early-onset presentation of cutaneous neurofibromas, cognitive abnormalities, somatic overgrowth, and dysmorphic facial features $(21,22)$. The c.2970-2972delAAT variant does not cause cutaneous or surface plexiform neurofibromas (23). Any of the C.5425C>T or C. $5425 C>A$ or C. $5425 C>C$ variants are related with café-au-lait spots, learning disabilities, short stature, and pulmonic stenosis but not cutaneous neurofibromas $(24,25)$. However, C16 (13 years of age) with the c.29702972del variant had neurofibromas, which is a late-onset feature of NF1.

A novel c.1637dupT variation was detected in C85 (20 years of age) who had isolated pilocytic astrocytoma without cutaneous findings. The case of $\mathrm{C} 11$ with the $\mathrm{c} \cdot 1721+3 \mathrm{~A}>\mathrm{C}$ variation only had clinical signs of neurofibromas without the accompanying café-au-lait spots. Ocular manifestations such as Lisch nodules and optic nerve glioma were determined in $23 \%$ and $6.8 \%$ of all cases, which are lower frequencies in comparison with the literature $(26,27)$.
Additionally, the frequency of neurofibromas were lower than in the literature.

Mosaic NF1 variants cause mild forms of the NF1 phenotype (28). We detected two different variations with low variation fraction in C87 (c.3721C>T) and C90 (c.5107C $>$ T). Sanger confirmations of these variants were consistent with NGS data. However, a molecular analysis of a second tissue could not be performed in these patients. Both of these patients had classical NF1 symptoms without family history and segmental involvement. We classified both of these patients as mosaic generalized NF1.

\section{Conclusion}

In conclusion, NF1 genetic analysis was a supporting tool for the atypical presentation of NF1 cases especially in the prepubertal period. Additionally, genetic analysis before pregnancy provides preimplantation and prenatal genetic diagnosis for families with NF1.

\section{Ethics}

Ethics Committee Approval: The study was approved by the Ethical Committee of the Gaziantep University Medical Faculty (approval number: 65587614-774.99-291, date: 04/10/2017). 
Informed Consent: Peripheral blood samples were obtained after taking informed consents from all participants or legal guardians of children under the age of 18.

Peer-review: Externally peer-reviewed.

\section{Authorship Contributions}

Concept: E.K., Design: E.K., Data Collection or Processing: H.M.A., E.K., Analysis or Interpretation: H.M.A., E.K., Literature Search: H.M.A., E.K., Writing: H.M.A., E.K.

Conflict of Interest: No conflict of interest was declared by the authors.

Financial Disclosure: The authors declared that this study received no financial support.

\section{References}

1. Lammert M, Friedman JM, Kluwe L, Mautner VF. Prevalence of neurofibromatosis 1 in German children at elementary school enrollment. Arch D ermatol 2005; 141:71-4.

2. Evans DG, Howard E, Giblin C, et al. Birth incidence and prevalence of tumor-prone syndromes: estimates from a UK family genetic register service. Am / Med Genet A 2010; 152A:327-32.

3. Adam MP, Ardinger HH, Pagon RA, et al. GeneReviews ${ }^{\circledR}$ [Internet]. Seattle (WA): University of Washington, Seattle; 1993-2021.

4. Williams VC, Lucas J, Babcock MA, Gutmann DH, Korf B, Maria BL. Neurofibromatosis type 1 revisited. Pediatrics 2009; 123:12433.

5. No authors listed. National Institutes of Health Consensus Development Conference Statement: neurofibromatosis. Bethesda, Md., USA, July 13-15, 1987. Neurofibromatosis 1988; 1:172-8.

6. Maruoka R, Takenouchi T, Torii C, et al. The use of next-generation sequencing in molecular diagnosis of neurofibromatosis type 1 a validation study. Genet Test Mol Biomark 2014; 18:722-35.

7. Stenson PD, Mort M, Ball EV, Shaw K, Phillips A, Cooper DN. The Human Gene Mutation Database: building a comprehensive mutation repository for clinical and molecular genetics, diagnostic testing and personalized genomic medicine. Hum Genet 2014; 133:1-9.

8. Richards S, Aziz N, Bale S, et al. Standards and guidelines for the interpretation of sequence variants: a joint consensus recommendation of the American College of Medical Genetics and Genomics and the Association for Molecular Pathology. Genet Med 2015; 17:405-24.

9. Cimino PJ, Gutmann DH. Neurofibromatosis type 1. Handb Clin Neurol 2018; 148:799-811.

10. Balla B, Árvai K, Horváth P, et al. Fast and robust nextgeneration sequencing technique using ion torrent personal genome machine for the screening of neurofibromatosis type 1 (NF1) gene. J Mol Neurosci 2014; 53:204-10.

11. Messiaen LM, Callens T, Mortier G, et al. Exhaustive mutation analysis of the NF1 gene allows identification of $95 \%$ of mutations and reveals a high frequency of unusual splicing defects. Hum Mutat 2000; 15:541-55.
12. Bianchessi $\mathrm{D}$, Morosini $\mathrm{S}$, Saletti $\mathrm{V}$, et al. 126 novel mutations in Italian patients with neurofibromatosis type 1. Mol Genet Genomic Med 2015; 3:513-25.

13. van Minkelen $\mathrm{R}$, van Bever $\mathrm{Y}$, Kromosoeto JN, et al. A clinical and genetic overview of 18 years neurofibromatosis type 1 molecular diagnostics in the Netherlands. Clin Genet 2014; 85:318-27.

14. Cawthon RM, Weiss $R, X u G F$, et al. A major segment of the neurofibromatosis type 1 gene: cDNA sequence, genomic structure, and point mutations. Cell 1990; 62:193-201.

15. Nemethova $M$, Bolcekova A, llencikova $D$, et al. Thirty-nine novel neurofibromatosis 1 (NF1) gene mutations identified in Slovak patients. Ann Hum Genet 2013; 77:364-79.

16. Ko JM, Sohn YB, Jeong SY, Kim HJ, Messiaen LM. Mutation spectrum of NF1 and clinical characteristics in 78 Korean patients with neurofibromatosis type 1. Pediatr Neurol 2013; 48:447-53.

17. Sabbagh A, Pasmant E, Imbard A, et al. NF1 molecular characterization and neurofibromatosis type I genotypephenotype correlation: the French experience. Hum Mutat 2013; 34:1510-8.

18. Zhang J, Tong $\mathrm{H}$, Fu $\mathrm{X}$, et al. Molecular Characterization of NF1 and Neurofibromatosis Type 1 Genotype-Phenotype Correlations in a Chinese Population. Sci Rep 2015; 5:11291. doi: 10.1038/srep11291.

19. Barrea C, Vaessen S, Bulk S, Harvengt J, Misson JP. Phenotypegenotype correlation in children with neurofibromatosis type 1. Neuropediatrics 2018;49:180-4.

20. Mautner VF, Kluwe L, Friedrich RE, et al. Clinical characterisation of 29 neurofibromatosis type-1 patients with molecularly ascertained 1.4 Mb type-1 NF1 deletions. I Med Genet 2010; 47:623-30.

21. Pasmant E, Sabbagh A, Spurlock G, et al. NF1 microdeletions in neurofibromatosis type 1: from genotype to phenotype. Hum Mutat 2010; 31:E1506-18. doi: 10.1002/humu.21271.

22. Upadhyaya $M$, Huson SM, Davies $M$, et al. An absence of cutaneous neurofibromas associated with a 3-bp inframe deletion in exon 17 of the NF1 gene (c.2970-2972 delAAT): evidence of a clinically significant NF1 genotype-phenotype correlation. Am J Hum Genet 2007; 80:140-51.

23. Pinna V, Lanari V, Daniele $P$, et al. p.Arg1809Cys substitution in neurofibromin is associated with a distinctive NF1 phenotype without neurofibromas. Eur J Hum Genet 2015; 23:1068-71.

24. Rojnueangnit $\mathrm{K}, \mathrm{Xie}$ ), Gomes $\mathrm{A}$, et al. high incidence of noonan syndrome features including short stature and pulmonic stenosis in patients carrying nf1 missense mutations affecting p.arg1809: genotype-phenotype correlation. Hum Mutat 2015; 36:1052-63.

25. Hirbe AC, Gutmann DH. Neurofibromatosis type 1: a multidisciplinary approach to care. Lancet Neurol 2014; 13:83443.

26. Ragge NK, Falk RE, Cohen WE, Murphree AL. Images of Lisch nodules across the spectrum. Eye (Lond) 1993; 7:95-101.

27. Jouhilahti EM, Peltonen S, Heape AM, Peltonen J. The pathoetiology of neurofibromatosis 1. Am / Pathol 2011; 178:1932-9.

28. Fisher MJ, Belzberg AJ, de Blank P, et al. 2016 Children's Tumor Foundation conference on neurofibromatosis type 1, neurofibromatosis type 2, and schwannomatosis. Am / Med Genet A 2017; 176:1258-69. 